\title{
High-efficiency Collimation of Airborne Sound through a Single Deep- subwavelength Aperture in an Ultra-thin Planar Plate
}

\author{
Jie $\mathrm{Hu}^{1,2, *}$, Bin Liang ${ }^{1}$, and Xiaojun Qiu ${ }^{3}$ \\ ${ }^{1}$ Key Laboratory of Modern Acoustics (MOE), Institute of Acoustics, Nanjing University, Nanjing, China \\ ${ }^{2}$ College of information Science and Technology, Nanjing Forestry University, Nanjing, China \\ ${ }^{3}$ Centre for Audio, Acoustics and Vibration, Faculty of Engineering and IT, University of Technology Sydney, Sydney, \\ Australia
}

\begin{abstract}
We propose a mechanism for high-efficiency collimation of airborne sound through an ultra-thin planar structure perforated with a single deep-subwavelength aperture by combining a zigzag-shaped structure with arrays of subwavelength resonators to increase the equivalent refractive index and eliminate high order diffractive waves simultaneously. Numerical simulations demonstrate the proposed mechanism enables remarkably-enhanced transmission and strong collimation of the transmitted wave which propagates for a strikingly-long distance exceeding 125 wavelength. The capability and flexibility of the proposed design opens up possibilities for novel compact acoustic-steering devices and may have far-reaching impacts in diverse applications such as acoustic communications and loudspeaker design.
\end{abstract}

Focusing and collimation of sound is of fundamental importance in acoustics with wide application potentials in various fields such as sonar communication, loudspeaker design, medical ultrasound imaging and therapy. Considering the long wavelength of acoustic waves used in practical situations, it would be highly desirable to produce collimated sound beam with compact structures for the sake of downscaling acoustic devices. The potentials of traditional methods for acoustic collimation that usually rely on active phased array technique are hampered by the complexity and high cost of the built array comprising a large number of transducers that need to be controlled separately by complicated electronic circuits [1-3]. The recent advent of acoustic artificial materials open up possibility for manipulating acoustic waves, enabling various fascinating wave-steering phenomena [4-11], especially effective spatial focusing and collimation of acoustic energy by using simple and low-cost passive structures [12-15].

In comparison to the bulky structures such as phononic crystals[16, 17] or perforated plates[18-20], acoustic metasurfaces proposed in the past few years offers a platform for producing special sound beams

\footnotetext{
*Email: hujie@njfu.edu.cn
} 
with subwavelength dimensions. However, the basic underlying mechanism of metasurface is based on synthesizing the desired wavefront by utilizing a line of point sources with different inital phases along the interface, which has to be implemented by a thin planar structure containing an array of openings for airborne sound such as space-coiling [21, 22] or cavity-and-pipe [23] types of metastructures. As we all know, the acoustic waves emit from a small hole generally radiates in all directions and the Fabry-Perotresonance-based enhancement of transmission is unattainable with deep-subwavelength thickness. So that it still remains challenging to produce a collimated sound beam through a single subwavelength aperture perforated on an ultra-thin plate despite the practical significance to diverse applications.

In this work, we propose a mechanism for breaking through the above limit and realize a collimated sound beam with high efficiency by using a planar rigid plate of deep-subwavelength thickness, perforated with a single aperture much thinner than the working wavelength. The aperture is designed to have a zigzag-shaped cross section for increasing the propagation distance of incident acoustic wave substantially, mimicking a straight slit filled with a high refractive index medium and enabling effective enhancement of transmission at relatively low frequency regime. On the other hand, the surface of the plate is rationally engineered to modify the radiation pattern on the transmitted side. We illustrate that the interference between the scattered wave from an array of subwavelength resonators decorated on the plate and the transmitted wave diffracted by the small aperture efficiently eliminates the high order diffracted waves, giving rise to strong collimation of acoustic wave. We verify our proposed mechanism via numerical simulations and demonstrate the performance of the designed device to realize strong acoustic directivity at low frequency and a collimated sound beam that propagates for more than 125 wavelength.

The schematic illustration of the proposed structure is shown in Fig. 1. For simplicity without loss of generality, we focus our attention on two dimensional (2D) systems in the current study. As shown in Fig. 1 , the whole structure is made of rigid materials that is completely impenetrable to airborne sound which can be easily fabricated by using different kinds of solids due to the huge contrast between the acoustic impedances of air and solids, except for a narrow slit that is located in the central region and has a deep subwavelength width at least an order of magnitude smaller than the working wavelength. In what follows we will explain our design idea and the proposed mechanism of giving rise to the desired highefficiency collimation of low frequency sound by using such an ultra-thin planar device perforated with a single deep-subwavelength aperture. 


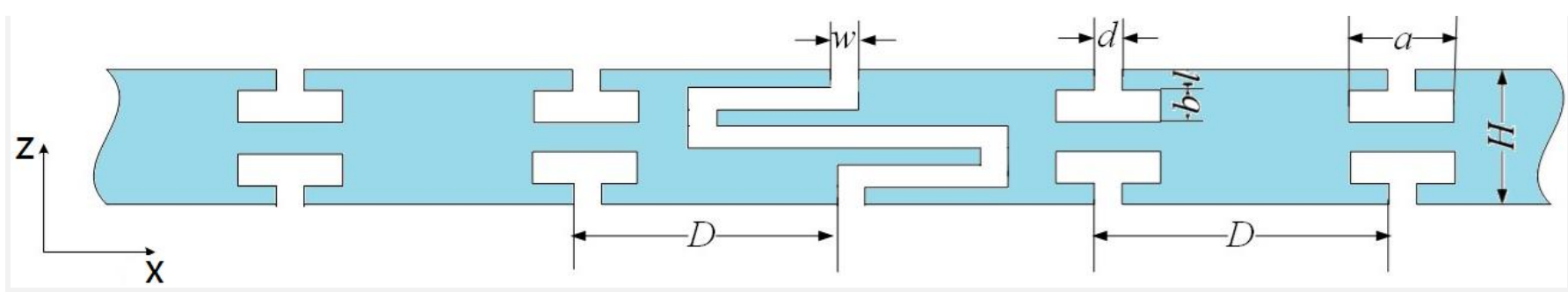

Figure 1. The schematic diagram of the proposed 2D ultra-thin planar structure for producing the high efficiency collimated acoustic beam transmitting through the zigzag aperture in the center.

Conventionally, the transmission efficiency of a plane acoustic wave that propagates along the positive $\mathrm{z}$ direction and impinges on such a structure normally is trivial due to the huge impedance mismatch induced by the extremely abrupt discontinuity in the cross-section. Only when appropriate acoustic resonances are excited in the structure can we achieve a substantial augment of acoustic intensity in the narrow region and a subsequent enhancement of transmission of acoustic energy through the deep-subwavelength aperture. However, as shown in Fig. 2(a), the Fabry-Perot type resonances supported by narrow channels are closely related to the working wavelength and need to occur at the specific frequencies that satisfy the condition $k_{0} L_{0}=m \pi(m=1,2, \ldots)$ where $L_{0}$ is the thickness of structure and $k_{0}=2 \pi / \lambda$ with $\lambda$ being the working wavelength. It is apparent that the thickness of the plate should be at least one half of wavelength to support the lowest F-P resonance mode, which is unattainable in systems of deep-subwavelength dimension in the propagation direction and substantially hinders the downscaling of the resulting devices.

To break through this limit, we propose to fill the slit located at the center with effective medium of high relative refractive index $n_{\mathrm{r}}$, which corresponds to a very low acoustic velocity $n_{\mathrm{r}} / c_{0}$ with $c_{0}=343 \mathrm{~m} / \mathrm{s}$ being the acoustic velocity in air, as shown in Fig. 2(b). Then the condition for the occurrence of FP resonance shifts to lower frequency regime, which makes it possible to achieve the extraordinary acoustic transmission in systems of deep-subwavelength dimension as the value of $n_{\mathrm{r}}$ is sufficiently large. As a practical implementation of this ideal model, we design a coiled channel that has deep-subwavelength width and a zigzag-shaped cross section for connecting the two openings on the two opposite sides of the proposed structure as shown in Fig. 2(c) which maintains the same ultra-thin thickness as Fig. 2(b). Due to the fact that acoustic waves, as scalar waves, propagate in a subwavelength channel under the cut-off frequency, such a space-coiling-type configuration forces the incident acoustic wave to travel along a path much longer than its physical dimension [5, 24]. As a result, the substantial elongation of the propagation distance of incident waves induces an extra phase delay to the transmitted wave and thereby remarkably increases the equivalent refractive index in the region in between. Apparently, the equivalent refractive 
index $n_{\mathrm{r}}$ in Fig. 1 can be calculated directly by relating to the total length of the coiled channel $L$ via $n_{\mathrm{r}}=$ $n_{0} L / H$, with $n_{0}$ being the refractive index of the surrounding air, so that $n_{\mathrm{r}}$ can be conveniently controlled by tailoring the number of coils. As a result, the thickness of the device is kept at deep-subwavelength scale, which is designed as $H=2 \mathrm{~mm}$, in stark contrast to the working wavelength.

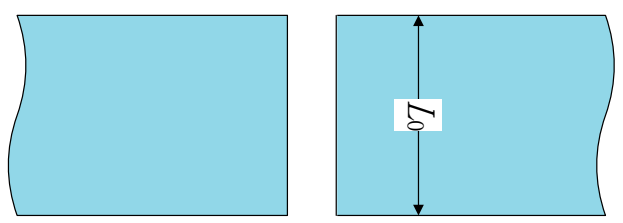

(a)

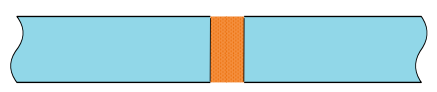

(b)

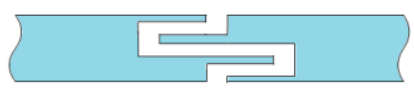

(c)

Figure 2. The schematic diagrams of three different structures for supporting F-P resonance at the same frequency: (a) thick plate perforated with a straight half-wavelength aperture;(b) ultra-thin plate with a straight aperture filled with high refractive index material (highlighted by orange square);(c) ultrathin plate with a zigzag aperture.

By designing a zigzagged-shaped structure to remarkably augment the effective refractive index in the central region, we excite strong FP resonance in the proposed structure of deep-subwavelength thickness and enable enhancement of transmission efficiency of incident acoustic energy through the extremely narrow slit that would otherwise virtually block the transmitted wave. This, however, is not sufficient for ensuring directional emission of outgoing acoustic wave on the transmitted side given the fact that such a slit will obviously diffract the transmitted acoustic energy into all directions due to its vanishing width in terms of the working wavelength. Then we engineer the surfaces of the proposed structure and design a periodic array for each side that consists of a series of identical Helmholtz-like cavities as shown in Fig. 1, with each having deep-subwavelength sizes in both two dimensions that helps to reduce the thickness of the resulting device. The resonant frequency of the 2-D Helmholtz resonators is

$$
f_{0}=\frac{c_{0}}{2 \pi} \sqrt{\frac{d}{(l+0.8 d) a b}},
$$

where $c_{0}=343 \mathrm{~m} / \mathrm{s}$ is the acoustic velocity in air, and the definitions of $a, b, d$ and $l$ are shown in Fig. 1.

Acoustic surface waves can be found at the interface of a periodically corrugating rigid device and air, whose dispersion is dominated by geometrical parameters of the perforated surface, such as the distance between each groove $D$. Incident waves are collected by acoustic surface waves instead of dispersion and funneling through the central aperture at a wavelength or half-wavelength close to $D$ [13]. With the coupling of waveguide at the interface and F-P resonance in the central aperture, a resonant peak 
appears.

In the current design, the total length and width of central aperture are $L=14.5 \mathrm{~mm}$ and $w=0.5$ $\mathrm{mm}$ respectively and a periodic arrays of $10 \mathrm{HR}$-like cavities in either side decorate the surface of device. The parameters of each cavity are $d=0.025 \mathrm{~mm}, l=0.4 \mathrm{~mm}, a=3.4 \mathrm{~mm}$ and $b=0.4 \mathrm{~mm}$, and the resonant frequency of each cavity is calculated as $11.42 \mathrm{kHz}$, which coincides with the working frequency. The distance between neighboring resonators is chosen as $D=14.5 \mathrm{~mm}$, nearly half of the incident working wavelength. In such cases, the interaction between the Helmholtz-like resonator and the incident acoustic wave is maximized owing to the strong scattering effect by the cavities at resonance, which enables effective coupling between the incident wave and the surface decorated with an array of such subwavelength resonators. As a result, the high orders of diffracted waves generated by the deep-subwavelength aperture are allowed to propagate on the surface of the proposed structure as special types of surface acoustic waves. This is similar to the wave process observed in planar structures whose surface is decorated with periodic groove arrays with half-wavelength or wavelength intervals, which also support propagation modes on the surface that stem from high diffracted modes but need the thickness of the whole structure to be comparable with the incident wavelength [13, 14]. Our mechanism uses subwavelength resonator arrays to realize conversion of high order modes to surface modes, leading to virtually elimination of diffraction effect on the transmitted wave and subsequent high-efficiency collimation of outgoing beam by an ultrathin planar structure.

We perform a series of numerical simulations to verify the effectiveness of the proposed mechanism. Throughout the manuscript, the numerical simulations are performed by using the finite element method based on COMSOL Multiphysics software. Figures 3 and 4 show the spatial distribution of acoustic field on the transmitted side and the typical results of the transmission of the designed structure as a function of frequency. The numerical results agree quite well with our theoretical predictions, revealing a substantial enhancement of transmission by the strong coupling effect between the FP resonance and the surface acoustic waves, as evidence by the near-unity transmission at $10.8 \mathrm{kHz}$. In contrast, extremely low transmission efficiency is observed for the rest frequencies as expected due to the lack of requisite resonance effect as shown in Fig 4. In our proposed mechanism, the resonance frequencies of the narrow aperture and the Helmholtz-like cavity need to be consistent, and the periodicity of the subwavelength cavity array must be equal to half of the incident wavelength, such that the acoustic energy of incident plane wave can be redistributed on the bottom side of structure (viz., the side on the plane of $\mathrm{z}=0$ as shown in Fig. 1) due to its interaction with the resonator array and then penetrates into the narrow slit despite the huge contrast in cross section. When there exists discrepancy between these parameters, the 
deviation of the incident frequency from the resonance frequencies will break this critical condition for effectively coupling the incident acoustic energy to the opposite side of structure, which have been proven via numerical simulations.

On the transmitted side, the radiated field emitted from the central aperture at frequency of $10.8 \mathrm{kHz}$ is depicted in Fig. 3. In comparison to the conventional case where the incident acoustic wave is diffracted by the narrow slit while passing through it and then radiates the acoustic energy to all directions, here the high-order modes of diffracted waves are virtually eliminated by the resonator arrays on the top side of structure (viz., the side on the plane of $\mathrm{z}>H$ as shown in Fig. 1) that uses the interaction with the FP resonances in the slit to convert efficiently the high order diffraction into surface acoustic waves and then prevents them from leaking to the far-field region. This is evident by the apparent directional emission of acoustic wave transmitted through the central aperture as clearly shown in the numerical results given by Fig. 3. Remarkably, after passing through a narrow aperture with subwavelength thickness with nearly 100 percent incident acoustic energy, the transmitted wave becomes a highly collimated beam that propagates in free space for a strikingly long distance exceeding 125 wavelength with no need to rely on complicated active phased array or artificial passive structures with bulky size or multiple openings, which would be highly desired in a variety of practical applications calling for directional focusing and emission of low-frequency sound such as the design of loudspeakers or sonar systems.

For quantitatively evaluating the radiation property of the proposed structure, we calculate the acoustic field in the far field and plot the typical results of the dependence of far field distribution of sound pressure on the azimuthal angle in the polar diagram shown in Fig. 5(a).

Emission of low-frequency acoustic energy with high directivity is of fundamental interests and practical importance in the field of acoustics. Yet the conventional methods have to rely on modulating curved surface, leaving the mechanism for effectively collimating acoustic waves absent which would be highly desired in diverse applications. The angular distribution of the emitted field by a rigid plate that has equal thickness and is perforated with a narrow aperture of the same width is also calculated and plotted for comparison as shown in Fig 5(b). It can be observed that in stark contrast to the reference plate that radiates the acoustic energy equally to different angles like a point source and accordingly has an almost omnidirectional radiation pattern, while our designed structure exhibits a strong directivity and is capable to limit the acoustic radiation with a narrow angle of approximately 25 degree. The conversion of a simple plane wave into highly collimated beam with sharp angular distribution by using a planar rigid plate with vanishing thickness and nearly seamless configuration is of particular practical significance to many important applications such as design of novel transducers for audible and 
underwater sound, which are in pursuit of miniaturization and integration of functional devices with the smallest possible openings yet capable to effectively emit directional acoustic beams propagating for a long distance.

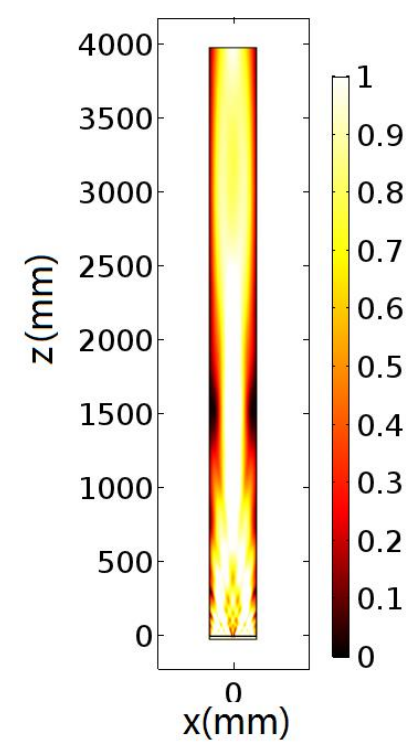

Figure 3. The simulated spatial distribution of the acoustic intensity on the transmitted side of the designed ultrathin planar plate with a deep-subwavelength aperture in the center as illuminated by a plane acoustic wave with a frequency of $10.8 \mathrm{kHz}$.

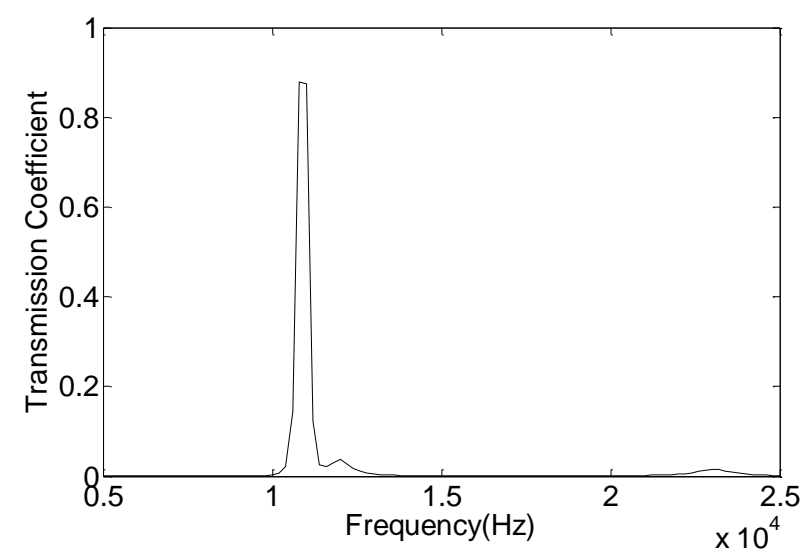

Fig 4. Simulated frequency dependence of the transmission coefficient of the designed device. 


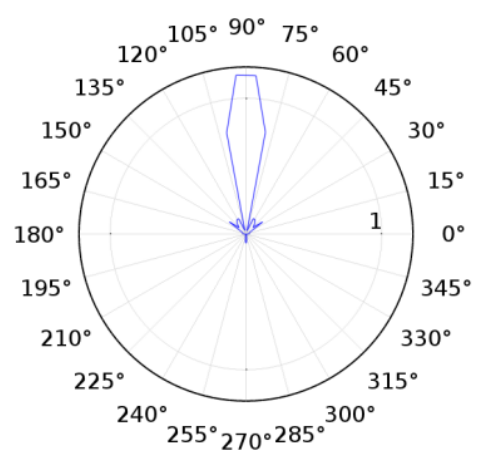

(a)

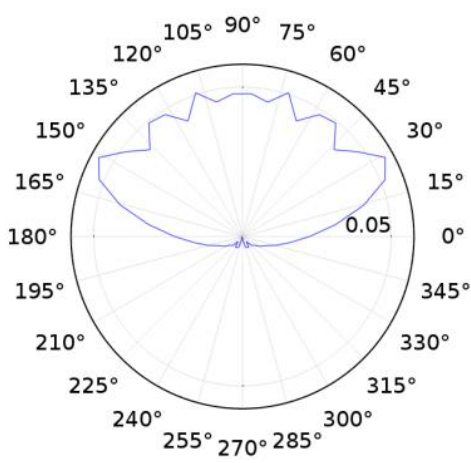

(b)

Figure 5. The simulated polar diagram of the far field sound pressure for (a) of the proposed device and (b) a same planar plate without the HR arrays on both sides at $10.8 \mathrm{kHz}$

\section{Conclusions}

In conclusion, we propose to generate high-efficiency collimation of airborne sound through an ultra-thin planar structure perforated with a single deep-subwavelength aperture, and demonstrate the metamaterial-based implementation of our design. The mechanism is based on the combination of a zigzag-shaped narrow channel located at the center and arrays of subwavelength resonators decorated on both sides, which simultaneously leads to substantial increase of the equivalent refractive index in the aperture and elimination of high order diffractive waves. This enables enhanced transmission and subsequent strong collimation of transmitted acoustic wave through the deep-subwavelength aperture that is conventionally unable to support FP resonance and diffracts the incident energy into all directions. The effectiveness of our proposed mechanism is verified via numerical simulations which demonstrate the high directivity of transmitted acoustic wave that propagates for a strikingly long distance exceeding 125 wavelength. With the capability of producing strong directivity at low frequency regime, our design opens route to unconventional manipulation of acoustic waves with compact dimensions and may have far-reaching impacts in versatile braches of acoustics ranging from acoustic communications to acoustic imaging.

\section{Acknowledgements}

This work was supported by the National Natural Science Foundation of China (Grant No. 11634006).

\section{References}

1. Yamada, M., N. Itsuki, and Y. Kinouchi, Adaptive directivity control of speaker array. 2005. 1443-1448 Vol. 2.

2. Woon-Seng, G., et al., A digital beamsteerer for difference frequency in a parametric array. IEEE Transactions on Audio, Speech, and Language Processing, 2006. 14(3): p. 1018-1025.

3. Olszewski, D., Steerable highly directional audio beamloudspeaker. Interspeech, 2005: p. 137-140.

4. Fang, N., et al., Ultrasonic metamaterials with negative modulus. Nature Materials, 2006. 5: p. 452.

5. Li, Y., et al., Extraordinary acoustic transmission through ultrathin acoustic metamaterials by coiling up space. Applied Physics Letters, 
2013. 103(6).

6. Akozbek, N., et al., Manipulating the extraordinary acoustic transmission through metamaterial-based acoustic band gap structures. Applied Physics Letters, 2014. 104(16).

7. Zhu, J., et al., A holey-structured metamaterial for acoustic deep-subwavelength imaging. Nature Physics, 2010. 7: p. 52.

8. Li, K., et al., Observation of unidirectional negative refraction in an acoustic metafluid prism. Applied Physics Letters, 2014. 104(4): p. 043505.

9. Yong, L., Q. Shuibao, and M.B. Assouar, Theory of metascreen-based acoustic passive phased array. New Journal of Physics, 2016. 18(4): p. 043024.

10. Jun, M. and W. Ying, Controllable transmission and total reflection through an impedance-matched acoustic metasurface. New Journal of Physics, 2014. 16(12): p. 123007.

11. Li, X.F., et al., Tunable Unidirectional Sound Propagation through a Sonic-Crystal-Based Acoustic Diode. Physical Review Letters, 2011. 106(8).

12. Zhu, Y.-F., et al., Multi-frequency acoustic metasurface for extraordinary reflection and sound focusing. AIP Advances, 2016. 6(12): $\mathrm{p}$. 121702.

13. Christensen, J., et al., Collimation of sound assisted by acoustic surface waves, in Nature Physics2007. p. 851-852.

14. Zhou, Y., et al., Acoustic Surface Evanescent Wave and its Dominant Contribution to Extraordinary Acoustic Transmission and Collimation of Sound. Physical Review Letters, 2010. 104(16).

15. Mei, J., et al., Acoustic wave transmission through a bull's eye structure. Applied Physics Letters, 2008. 92(12): p. 124106.

16. Picó, R., et al., Spatial filtering of sound beams by sonic crystals. Applied Acoustics, 2012. 73(4): p. 302-306.

17. Liu, F., et al., Tunable transmission spectra of acoustic waves through double phononic crystal slabs. Applied Physics Letters, 2008. 92(10): p. 103504.

18. Estrada, H., et al., Sound transmission through perforated plates with subwavelength hole arrays: A rigid-solid model. Wave Motion, 2011. 48(3): p. 235-242.

19. Estrada, H., et al., Extraordinary Sound Screening in Perforated Plates. Physical Review Letters, 2008. 101(8): p. 084302.

20. Zhou, L. and G.A. Kriegsmann, Complete transmission through a periodically perforated rigid slab. Journal of the Acoustical Society of America, 2007. 121(6): p. 3288.

21. Li, Y., et al., Reflected wavefront manipulation based on ultrathin planar acoustic metasurfaces. Scientific Reports, 2013. 3(1): p. 25462546.

22. Li, Y., et al., Experimental Realization of Full Control of Reflected Waves with Subwavelength Acoustic Metasurfaces. Physical Review Applied, 2014. 2(6).

23. Li, Y., et al., Metascreen-Based Acoustic Passive Phased Array. Physical Review Applied, 2015. 4(2): p. 024003.

24. Liang, Z. and J. Li, Extreme Acoustic Metamaterial by Coiling Up Space. Physical Review Letters, 2012. 108(11): p. 114301. 